Article

\title{
A Novel Simple Anti-Ice Aluminum Coating: Synthesis and In-Lab Comparison with a Superhydrophobic Hierarchical Surface
}

\author{
Marcella Balordi ${ }^{1, *}$, Giorgio Santucci de Magistris ${ }^{1,2}$ and Cristina Chemelli ${ }^{3}$ (I) \\ 1 RSE, Ricerca sul Sistema Energetico, Strada Torre della Razza, 29122 Piacenza, Italy; \\ giorgio.santucci@rse-web.it \\ 2 Facoltà di Scienze Chimiche, Università Degli Studi di Pavia, viale Taramelli 12, 27100 Pavia, Italy \\ 3 RSE, Ricerca sul Sistema Energetico, via Rubattino 54, 20134 Milano, Italy; cristina.chemelli@rse-web.it \\ * Correspondence: marcella.balordi@rse-web.it
}

Received: 16 December 2019; Accepted: 25 January 2020; Published: 28 January 2020

\begin{abstract}
A simple process to obtain a slippery surface with anti-ice and ice-phobic properties has been developed and characterized in laboratory. The coating is realized by growing a nanostructured pseudo-boehmite on an aluminum substrate and applying an environmentally compatible final functionalization consisting of a fluorine-free oligomeric short-chain alkylfunctional silane. The resulting surface is conceptually similar to a slippery liquid infused porous surface (SLIP) material, but the porous infrastructure is inorganic and the process to generate it is very simple, rapid and economic. The coating performance in terms of hydrophobicity at room and low temperatures, ice nucleation temperature and ice adhesion forces were assessed in laboratory. Moreover, hydrophobicity persistence was tested after prolonged immersion in acid, basic and saline solutions while the durability of ice-phobic behavior was assessed by repeated shear stress tests. Results are compared with those of a superhydrophobic hierarchical aluminum surface obtained with a fluorinated siloxane. The novel coating shows very good anti-ice properties and relevant durability, with some differences from the fluorinated surface. The novel slippery coating process is promising for industrial applications, also taking into account its environmental compatibility, simplicity and low cost.
\end{abstract}

Keywords: superhydrophobic; slippery; ice-phobicity; durability; nanostructure; coating; aluminum

\section{Introduction}

Ice and snow accumulation can cause serious problems in many fields, for example aircrafts, roads and railways, wind turbines and photovoltaics [1]. The accretion of ice or snow sleeves on overhead power lines (OHL) may also cause the failure of conductors and guard wires and/or the collapse of the towers, provoking the disruption of the transmission networks and the interruption of the communication optical fibers embedded in the guard wires [2] with consequent severe economic impacts.

Recently, several superhydrophobic (SHP) coatings were studied for anti-icing applications: the excellent water repellency property sometimes leads to ice-phobicity; however, their application is still controversial [3-8]. Moreover, the durability aspects of SHP coatings and surfaces have to be taken into serious account. Many studies indeed evidence the quick deterioration of the SHP surfaces with loss of the hydrophobic and ice-phobic properties [8-10], while other works demonstrate that an accurate design of the SHP surfaces, based on a fine-tuning of both the morphology and the deposition method of the coating, gives rise to a real durable ice-phobic material [11]. However, the development of a 
scalable and industrially feasible process, for instance for the fabrication of cables for overhead power lines, is still an open issue.

In a previous work, we studied a superhydrophobic surface obtained by applying a commercial fluoroalkyl silane (FAS) on a hierarchical micro-nano aluminum structure [12]. This coating maintained hydrophobic behavior even at low temperatures and evidenced amazing durability of ice-phobic properties after several freeze-thaw cycles in shear stress tests; however, the durability of this coating exposed in harsh environmental conditions was to be fully understood. Moreover, despite the very quick process to produce the ice-phobic material and the good results in terms of the ice-phobic property and its durability, the presence of polyfluorinated alkyl molecules may strongly limit the application of this coating for environmental problems [13].

Another interesting field of study concerns the development of slippery liquid infused porous surfaces (SLIPS) coatings composed of a micro/nanoporous structure with low surface energy, infused with a lubricant fluid $[1,14,15]$. These materials are designed according to two principles: (1) the lubricating fluid and the water have to be immiscible; (2) the chemical affinity between the lubricating fluid and the solid should be higher than that between the water and the solid [16]. Thanks to the presence of the infused fluid, locked in the micro-nanostructure, SLIPSs evidence a very good capability in repelling various liquids, in keeping very low contact angle hysteresis and in quickly restoring the lubricant after mechanical abrasion or damages [17]. They are also considered perspective materials for anti-icing applications because of their impressive stability in high humidity conditions [15] and their ability in reducing the ice adhesion strengths [1].

However, the anti-icing durability of SLIPS coatings is still a challenge [18]; in particular, icing/deicing cycles, water droplets and low temperatures, which can accelerate the lubricant depletion $[19,20]$. Moreover, to obtain SLIPS, complicated and multi-step synthetic methods are necessary, employing expensive starting materials and technologies [21-24].

In this work, we present a novel aluminum slippery surface (SLS) that is synthesized by boiling the aluminum substrate to grow a nanostructured pseudo-boehmite and by applying an oligomeric short-chain fluorine-free alkyl siloxane. We studied its wettability under different environmental conditions and its ice-phobic behavior; eventually, we assessed the durability of its properties. This surface is characterized by a light hydrophilic static contact angle, a very low sliding angle and low ice adhesion. SLS has similar anti-icing, wettability and even better durability behavior of SLIPS, despite the considerable differences in the morphology and in the chemistry: indeed, the under-layer structure is a porous hydrophilic inorganic oxide, no lubricant is infused and the final surface is dry.

The process to obtain the SLS is very intriguing because it is fast, environmentally friendly, economical, no special equipment and expensive chemicals are needed and thus could be easily transferred to industrial production. Although the process to grow the pseudo-boehmite is well known [25] and it is also very often used to build a nanostructure, as far as we know, a full characterization of durability and ice-phobicity of a surface similar to SLS is still missing.

Results and performance in terms of hydrophobicity at room and low temperatures, ice adhesion forces and durability are compared with those of the superhydrophobic boehmite surface studied in our previous work [12]. This study aims to directly compare results obtained in the same laboratory, applying the same ice-phobic characterization methods and the same aging protocols, given that, so far, a recognized protocol to assess the durability of the coatings is missing. Some important results are evidenced, indicating that slippery surfaces better tolerate critical and aggressive environmental conditions; however, the SHP coating keeps higher durability of ice-phobic properties.

\section{Materials and Methods}

\subsection{Materials}

Flat plates $(20 \mathrm{~mm} \times 70 \mathrm{~mm} \times 2 \mathrm{~mm})$ and bars $(12 \mathrm{~mm}$ diameter $\times 100 \mathrm{~mm}$ length $)$ of aluminum alloy (6082) were used as substrates. 
Dynasylan ${ }^{\circledR}$ SIVO CLEAR EC was purchased from EVONIK (Essen, Germany); the product is composed of fluoroalkylsilane (FAS) $2 \%$, propan-2-ol 93\% and dodecane $5 \%$, and was used as received.

Dynasylan ${ }^{\circledR} 9896$ was purchased from EVONIK and it is an oligomeric short-chain alkylfunctional silane. It was used after dilution 1:1 in volume in ethanol.

Hexane $(95 \%)$, acetone $(>99.5 \%)$ and acetic acid $(\geq 99.7 \%)$ were purchased from Sigma Aldrich (St. Louis, MO, USA).

Ethanol ( $\geq 99.9 \%)$, ethylene glycol $(<98 \%)$, sodium chloride and sodium hydroxide were purchased from Carlo Erba (Milan, Italy).

Diiodomethane (99\%) was purchased from Alfa Aesar (Haverhill, MA, USA).

\subsection{Preparation of Superhydrophobic Surface (SHP)}

The aluminum alloy specimens were cleaned with basic soap, rinsed in an ultrasonic bath for $10 \mathrm{~min}$ with acetone and dried under nitrogen flux. For the preparation of the SHP samples, the aluminum alloy was sandblasted with micro glass beads in the 40-70 $\mu \mathrm{m}$ diameter range to generate a micrometric roughness. The sandblasting process was carried out for $10 \mathrm{~s}$ for each exposed area, with an applied pressure of 3.5 bar while keeping the sample $5 \mathrm{~cm}$ distant from the nozzle. Afterward, in order to generate a pseudo-boehmite nanostructured layer, the sample was boiled for $5 \mathrm{~min}$ at $100^{\circ} \mathrm{C}$ in ultrapure water. Then, it was dried in air, rinsed with n-hexane and dried under nitrogen flux, as described in $[12,26,27]$. The obtained hierarchical substrate was dip-coated in the FAS bath (dipping-withdrawing speed: $0.7 \mathrm{~mm} / \mathrm{s}$, permanence time: $120 \mathrm{~s}$ ) and cured at $70{ }^{\circ} \mathrm{C}$ for $1 \mathrm{~h}$.

\subsection{Preparation of Slippery Surface (SLS)}

For the preparation of the slippery surface, the aluminum alloy specimens were cleaned with basic soap, rinsed in an ultrasonic bath for $10 \mathrm{~min}$ with acetone and dried under nitrogen flux. To obtain a porous nanostructured pseudo-boehmite, the aluminum alloy was boiled for $30 \mathrm{~min}$ at $100{ }^{\circ} \mathrm{C}$ in ultrapure water, dried in air, rinsed with n-hexane and dried under nitrogen flux. Dynasilan ${ }^{\circledR} 9896$ was diluted in ethanol (1:1 in volume) and the solution (Dyn-50) was stirred for $1 \mathrm{~h}$. The aluminum samples were dip-coated in ALK (dipping-withdrawing speed: $0.7 \mathrm{~mm} / \mathrm{s}$, permanence time: $120 \mathrm{~s}$ ) and cured at $120^{\circ} \mathrm{C}$ for $1 \mathrm{~h}$.

\subsection{Characterization Methods}

Surface morphologies of all the samples and the thickness of the oxide layers were examined using a field emission scanning electron microscope (FE-SEM; Mira III, Tescan, Brno, Czech Republic). Energy dispersive X-ray spectroscopy (EDS) (Bruker, Billerica, MA, USA) micro-analysis was used to determine the composition of the materials.

A Taylor Hobson stylus profilometer was used to measure surface roughness. Data were averaged over at least 5 runs for each sample.

The Vickers hardness was estimated by the means of the FM 810E micro-hardness tester (Future-tech Corp., Kawasaki, Japan) applying a force of $1 \mathrm{~g}$ to the indenter. Data were averaged over 5 tests for each sample.

The FT-IR characterizations were conducted with the FT-IR Alpha 1 (Bruker) spectrometer with ATR apparatus (diamond crystal was used as the internal reflection element).

The gravimetric measure was performed with a microbalance Cubis MSU6 (Sartorius, Gottingen, Germany) with a sensitivity of $0.001 \mathrm{mg}$.

The static water contact angles (WCA) were measured with the DSA 30 Drop Shape Analyzer (Kruss, Hamburg, Germany) with the method of the sessile drop using $4 \mu \mathrm{L}$ volume of water at $20^{\circ} \mathrm{C}$ on at least 5 points for each sample. The WCA measurements at low temperatures were conducted by means of a Peltier plate in an environmental chamber at $-4{ }^{\circ} \mathrm{C}$, deposing the drop on the cooled samples.

The measurements of dynamic contact angles (hysteresis (CAH), roll-off (RA) and sliding angles (SA)) at $20^{\circ} \mathrm{C}$ were performed with the tilting plate method using $20 \mu \mathrm{L}$ volume of water. 
The measurements of the dynamic contact angles at $-4{ }^{\circ} \mathrm{C}$ were performed with the captive needle method in the Peltier chamber (Kruss, Hamburg, Germany). To measure the roll-off angles at $-4{ }^{\circ} \mathrm{C}$, the Peltier chamber was attached to a home-made tilting table and using a droplet of $20 \mu \mathrm{L}$ of water. Data were averaged on at least 3 measures for each sample.

The measurements of surface free energy (SFE) were performed with OWRK model measuring static contact angles with water, diiodomethane and ethylene glycol.

The ice nucleation temperature $T_{n}$ was measured in the following way: the sample was attached to a Peltier cell and covered by a transparent lid. A water droplet of $10 \mu \mathrm{L}$ was deposited at $20^{\circ} \mathrm{C}$ on the sample; then, the temperature was cooled down with a cooling rate of $6{ }^{\circ} \mathrm{C} / \mathrm{min}$ until the $\mathrm{T}_{\mathrm{n}}$ was reached and the drop started solidification [28].

The ice adhesion strength was evaluated by means of the shear force needed to extract a sample pole from an ice block. We adopted a home-made apparatus equipped with an electromechanical testing system (INSTRON 4507). The testing process and the apparatus are described in the Supplementary Materials section. No standard procedure was available for the ice-phobicity tests.

\subsection{Durability Performance Tests}

The durability study on the samples followed a protocol consisting of four tests (three in wet chemicals and one for ice shedding) and, for each test, different conditions were implemented according to $[12,24,29]$ Hydrophobic and/or ice-phobic properties of the samples were measured after each test phase and compared to the pristine sample. The test frequencies and applied procedure allow distinguishing the different aging behaviors of the samples. Further information about the durability protocol is reported in the Supplementary Materials.

\subsubsection{Resistance in Wet Chemicals}

Freshly prepared SLS and SHP samples were used for each test. Different solutions were prepared to assess the durability of each sample in three different conditions: acid (acetic acid, $\mathrm{pH}=3$ ), basic (sodium hydroxide, $\mathrm{pH}=8.5$ ) and saline (sodium chloride $35 \mathrm{~g} / \mathrm{L}$ ). The as-prepared solutions should assess the resistance in different conditions: the acid solution simulated the $\mathrm{pH}$ of an acid rain event, the alkali environment recreated the $\mathrm{pH}$ of the seawater and the ionic solution recreated an average sea salt concentration. Samples were completely immersed in this aging solution for different times then rinsed with water and dried under nitrogen. The characterization was done by measuring WCA, contact angle hysteresis (CAH) and RA/SA to assess the variation of hydrophobic properties. Five and three replicated measurements were performed for WCA and for CAH/RA/SA, respectively. Tests were conducted at room temperature to avoid the combination of multiple effects.

\subsubsection{Resistance to Ice Shedding}

The resistance of the surfaces to ice removal was tested by repeating the shear stress test on the same samples several times; ten bars for each sample were tested and the average shear stress was calculated. The variation of the shear force required to remove ice after several (up to 20) icing-deicing cycles allows assessing the durability of ice-phobic properties.

\section{Results and Discussion}

\subsection{Morphological and Chemical Evaluation}

The hierarchical surface of SHP is shown in Figure 1. At low magnification, the micrometric roughness generated by the sandblasting process is visible; the resulting roughness $\left(R_{\mathrm{a}}\right)$ is $0.94 \mu \mathrm{m}$. At higher magnification, the typical grass-like aspect of the pseudo-boehmite layer is observable [30]. A thickness of about $300 \mathrm{~nm}$ was estimated for the oxide layer [31]. 


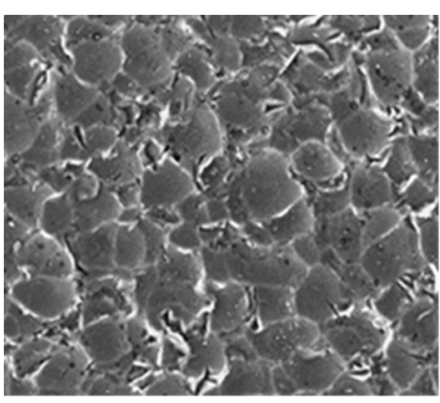

$100 \mu \mathrm{m}$

(a)

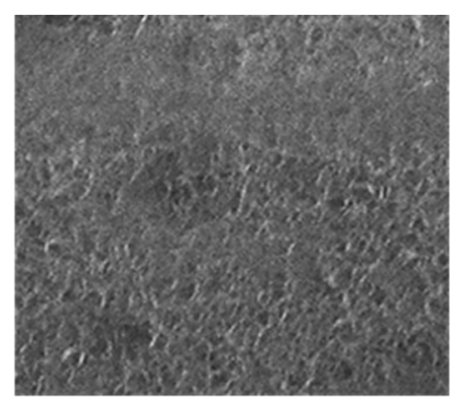

$1 \mu \mathrm{m}$

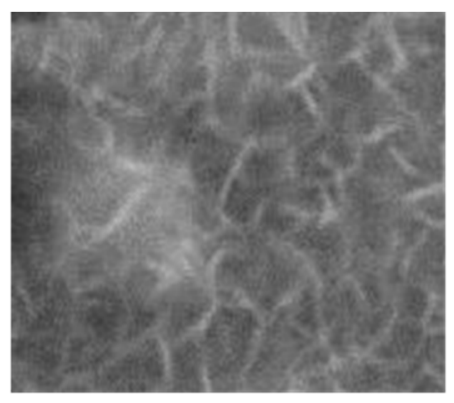

$500 \mathrm{~nm}$

(c)

Figure 1. SEM images of superhydrophobic (SHP) surface at different magnifications; (a) sandblasted micro-rough surface, secondary electron (SE) at 500×; (b) nanostructured pseudo-boehmite surface, SE, 20,000×; (c) nanostructured pseudo-boehmite surface, SE, 50,000×.

The presence of the FAS layer is not visible due to its extremely thin thickness, though it is revealed by the signal of fluorine in the EDX spectra (fluorine atomic concentration $2 \%$ ). The thickness of the FAS layer is about $1.5 \mathrm{~nm}$ according to [32].

The roughness $\left(R_{\mathrm{a}}\right)$ of the SLS aluminum substrate was $0.30 \mu \mathrm{m}$, and after the boiling process a boehmite layer of about $500 \mathrm{~nm}$ of thickness was obtained [31]. Differently than SHP, in the SEM images of SLS (Figure 2), the nano-grass-like pseudo-boehmite is partially hidden; only some pinnacles are visible. In fact, the Dyn-50 completely fills up the nanovoids in the pseudo-boehmite layer and the surface is almost totally covered by a much thicker layer than the FAS one. The thickness is calculated at about $1 \mu \mathrm{m}$ according to the gravimetric analysis and the Landau-Levich model [33].

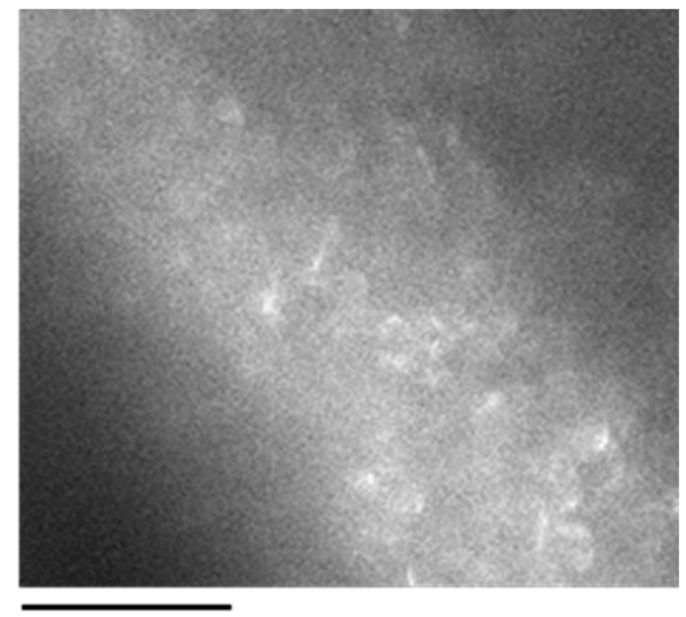

$1 \mu \mathrm{m}$

Figure 2. SEM images of the slippery surface (SLS) surface, SE, 20,000 $\times$.

In this sample, the role of the porous pseudo-boehmite is dual: firstly, similarly to SHP, it improves the adhesion of the coating thanks to Al-O-Si bonds, secondly, the porosity of the nano-oxide layer allows to host a large amount of Dyn-50. In fact, by comparing the EDX semi-quantitative analysis of the SLS sample with that of a non-nanostructured aluminum sample similarly covered with Dyn-50 (SLS-1), a silicon content (attributable to the Dyn-50) much higher in SLS than in SLS-1 can be appreciated (Table 1). 
Table 1. EDX atomic concentration in SLS and SLS-1 samples.

\begin{tabular}{ccccc}
\hline Sample & Si (at.\%) & Al (at.\%) & O (at.\%) & C (at.\%) \\
\hline SLS & 4 & 56 & 17 & 23 \\
SLS-1 & 1 & 67 & 8 & 24 \\
\hline
\end{tabular}

The FT-IR spectra (Figure 3) further confirm that SLS is hosting a bigger amount of Dyn-50 than SLS-1. This is well visible in the peaks at $2960-2872 \mathrm{~cm}^{-1}$, referable to the stretching mode of C-H bonds in the siloxane. In the SLS spectrum, the peaks related to the pseudo-boehmite layer are also visible at $780-470 \mathrm{~cm}^{-1}$ together with the weak peaks at 901 and $864 \mathrm{~cm}^{-1}$ attributable to the stretching of $\mathrm{Si}-\mathrm{O}-\mathrm{Al}$ bonds [34]. It is also important to underline the presence of the signals due to $\mathrm{Si}-\mathrm{O}-\mathrm{Si}$ $\left(1060 \mathrm{~cm}^{-1}\right)$ indicating the presence of siloxane chains.

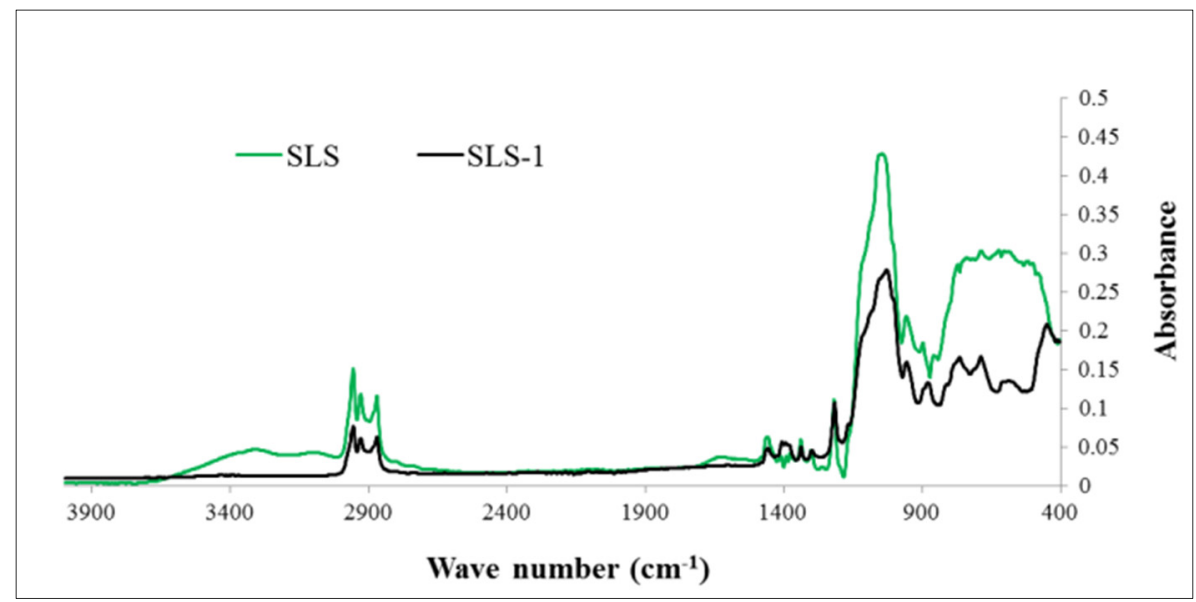

Figure 3. FT-IR spectra of SLS and SLS-1: $\left(2960-2872 \mathrm{~cm}^{-1}\right) \vee \mathrm{C}-\mathrm{H} ;\left(1463-1297 \mathrm{~cm}^{-1}\right) \omega \mathrm{C}-\mathrm{H}$; $\left(1215 \mathrm{~cm}^{-1}\right) \vee \mathrm{Si}-\mathrm{CH}_{2} ;\left(1060 \mathrm{~cm}^{-1}\right) \vee \mathrm{Si}-\mathrm{O}-\mathrm{Si}$.

Thanks to the gravimetric analysis, it was estimated that SLS hosts almost 70\% more of Dyn-50 than SLS-1 (about $149 \mu \mathrm{g} / \mathrm{cm}^{2}$ and $87 \mu \mathrm{g} / \mathrm{cm}^{2}$ of siloxane were calculated, respectively).

\subsection{Hydrophobic Properties at Room and Low Temperatures}

The static (WCA) and dynamic angles (CAH and RA/SA) of SHP and SLS were collected at room and low temperatures and are shown in Table 2 together with the surface energy data (SFE).

Table 2. Wettability and surface free energy at room and low temperatures.

\begin{tabular}{cccccccc}
\hline \multirow{2}{*}{ Sample } & \multicolumn{3}{c}{ Room Temperature } & \multicolumn{3}{c}{ Low Temperature } \\
\cline { 2 - 7 } & WCA $\left(^{\circ}\right)$ & CAH $\left({ }^{\circ}\right)$ & RA/SA $\left(^{\circ}\right)$ & SFE $\left(\mathbf{m J} / \mathbf{m}^{2}\right)$ & WCA $\left(^{\circ}\right)$ & CAH $\left(^{\circ}\right)$ & RA/SA $\left({ }^{\circ}\right)$ \\
\hline SHP & $170.2 \pm 2.7$ & $4.2 \pm 0.7$ & $2 \pm 1$ & $0.11 \pm 0.03$ & $143.2 \pm 2.7$ & $32.3 \pm 2.7$ & $>90$ \\
SLS & $86.7 \pm 1.5$ & $2.0 \pm 0.1$ & $2.0 \pm 0.2$ & $38.50 \pm 0.02$ & $81.5 \pm 0.9$ & $8.6 \pm 0.8$ & $16.0 \pm 2.0$ \\
\hline
\end{tabular}

The SHP sample evidences very good superhydrophobic properties with high WCA and very low RA and $\mathrm{CAH}$; however, at low temperatures, the surface loses its super-hydrophobicity and the dynamic properties highly degenerate. This behavior is typical of SHP surfaces [14] and it is mainly due to the humidity condensation from the surrounding air on the surface that drives the transition from the Cassie-Baxter to the Wenzel state [35].

The SLS evidences a weak hydrophilic behavior at room temperature, comparable with that of bare aluminum (WCA about $85^{\circ}$; SFE about $33 \mathrm{~mJ} / \mathrm{m}^{2}$ ) [36]. Despite this, the dynamic properties are consistently better with respect to the bare aluminum. The very low hysteresis and sliding angles are 
comparable to those of SLIPS [37] but, notwithstanding this similarity, SLS cannot be classifiable as a SLIPS. Indeed, the porous surface in SLS is composed of hydrophilic pseudo-boehmite while SLIPS typically have a hydrophobic porous structure, as previously discussed in [16]; moreover, the surface is dry and there is not a liquid intra-layer between the solid and the repellent fluid [38].

At low temperatures, the wettability tests of SLS evidence a little reduction of the static angle but the SA and the hysteresis keep low values. This behavior indicates that the SLS coating better tolerates the cold conditions and the humidity condensation phenomena, similarly to SLIPS coatings [37]. Probably, this is due to the absence of impurities and surface defects, which are very likely to be present on a rough superhydrophobic surface exposed to condensed humidity. On the smooth surface of SLS, the receding angle, which is the parameter related to the adhesion of the drops [39], keeps a good value, contributing to maintaining a low hysteresis even at low temperatures. As a matter of fact, the underlying pseudo-boehmite in SLS seems to have an important role in decreasing the hysteresis and sliding angle both at room and low temperatures: indeed, the un-boiled SLS-1 sample showed worse dynamic properties than SLS at room temperature (CAH 9.6 and SA $\left.12.3^{\circ}\right)$ and even worse at $-4{ }^{\circ} \mathrm{C}\left(\mathrm{CAH} 11.8\right.$ and SA $\left.26.3^{\circ}\right)$. Further investigation to explain this phenomenon will be done.

\subsection{Ice-Proof Performances}

Although a protocol to measure ice-proof performances is not defined yet, two main parameters can be studied to evaluate the properties of a surface with respect to the ice accretion and adhesion: the ice nucleation temperature $\left(T_{\mathrm{n}}\right)$ and the ice adhesion strength on the surface. The ability of a surface in lowering the $T_{\mathrm{n}}$ of a supercooled water droplet is defined in literature as anti-icing property [40]; thanks to this, the surface can prevent or hinder the ice formation. In parallel, the ability of the surface to lower the adhesion of the ice can be referred to as ice-phobicity and it defines how easily the ice can be removed from the surface [22].

Considering these definitions, we studied both anti-icing and ice-phobic properties of the coated samples compared to the bare aluminum ones.

\subsubsection{Anti-Icing Properties}

According to the literature [41], ice nucleation is a heterogeneous process in which ice nuclei forms at the interface with the solid substrate rather than inside the water droplet for energetic reasons. The heterogeneous nucleation is therefore strictly related to the underlying surface structure, and in particular to the presence of sites favoring the nucleation and to the contact area between water and solid surface. A method to measure the influence of the surface structure on the ice nucleation process is to analyze the temperature $T_{\mathrm{n}}$ at which the drop starts to solidify, as described above.

In our experimental setting, the water droplets on the bare aluminum froze at $-7.8^{\circ} \mathrm{C}$ while the $T_{\mathrm{n}}$ for SHP and SLS were -12.1 and $-12.4^{\circ} \mathrm{C}$, respectively (Figure 4 ).

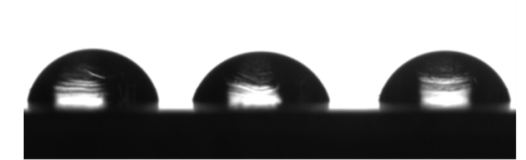

Bare aluminum at $20^{\circ} \mathrm{C}$

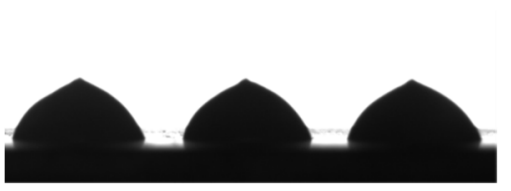

Bare aluminum at $-7.8^{\circ} \mathrm{C}$

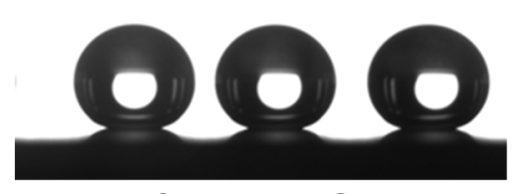

SHP at $20^{\circ} \mathrm{C}$

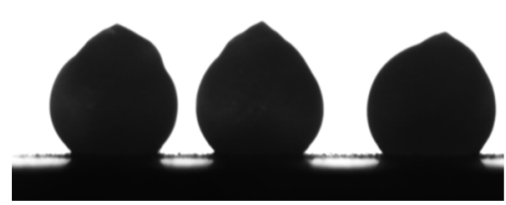

SHP at $-12.1^{\circ} \mathrm{C}$

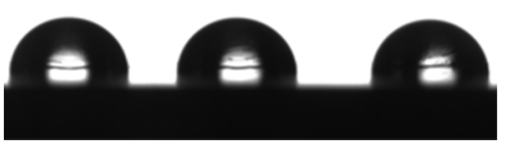

SLS at $20^{\circ} \mathrm{C}$

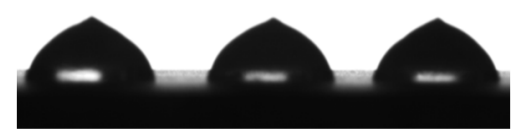

SLS at $-12.4{ }^{\circ} \mathrm{C}$

Figure 4. Icing nucleation temperature $T_{n}$ on different surfaces. 
The experimental results demonstrate that both the SHP and SLS surfaces can hinder the formation of frozen droplets, providing interesting and comparable anti-icing properties, although attributable to different mechanisms.

The efficacy of the superhydrophobic surface in reducing ice formation is related to the small contact area between the liquid and the solid. Indeed, the water droplet floats on the superhydrophobic surface and it comes into contact with this only for a small area. This leads to a reduction in both heat exchange and the ice nucleation rate [42-44]. Moreover, it is well reported that in SHP coatings, the air pockets further hinder the freezing process by acting as heat insulators [45,46], delaying the heat exchange.

The anti-icing behavior of SLS is comparable to the SHP in terms of Tn. Two main factors are to be considered. First, the surface is almost completely impregnated with the Dyn-50, which creates a heat insulation effect similar to the air pockets in an SHP surface [47]. Second, the presence of hydrogen bonds favors the ice nucleation and the SLS does not contain chemical groups capable of forming hydrogen bonds. Moreover, it is reported that the interaction between a hydrophilic surface and water can prevent the first layer of water molecules from arranging in ice structure [48].

\subsubsection{Ice-Phobic Properties}

The shear stress force values and the calculated adhesion reduction factor (ARF) are displayed in Figure 5. The ARFs were calculated as the ratio between the shear stress values of bare aluminum alloy and of the samples.

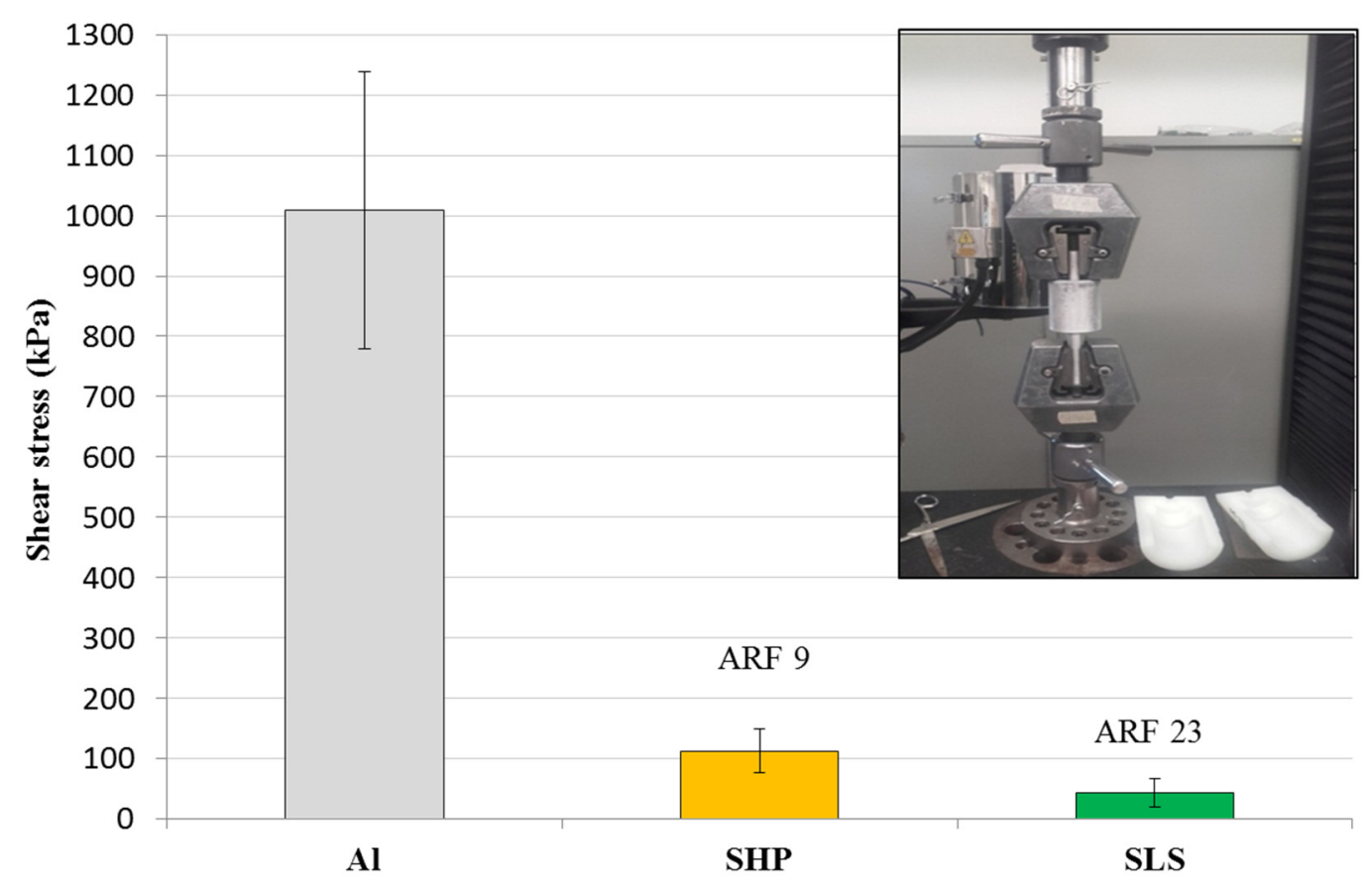

Figure 5. Shear stress values for bare aluminum, SHP and SLS samples (adhesion reduction factors are also reported). In the box, the home-made equipment used for shear stress measurements.

Both superhydrophobic and slippery surfaces evidenced a good reduction of ice adhesion with respect to the bare aluminum alloy; noteworthy, the SLS has better ice-phobic properties.

As described in several studies $[12,39,49,50]$, the ice-phobic behavior of the SHP surface can be attributed to the high water repellency and to the existence of an "air-cushion" on the surface of the sample that maintain it partially unwetted during the ice formation or that considerably reduce the contact area of ice on the surface. The ice-phobic results obtained for SHP are comparable or better than those of superhydrophobic materials reported in the literature [51-53]. 
The SLS evidenced an even higher ARF than the SHP. This remarkable result is comparable to the shear stress values of several SLIPS coatings [28,38,54,55].

On a molecular scale, the presence of the non-polar allylic groups completely covering the surface gives rise to very weak intermolecular attraction forces between surface and ice. Thus, the electrostatic van der Waals forces and the hydrogen bonds, between ice and the sample, contribute little to the ice adhesion, minimizing the shear force $[51,56]$. On a microscopic scale, the SLS is a smooth surface because the nanoporous layer is filled with the coating: this leads to a minor surface area in contact with the ice and, consequently, to a decreasing of the ice adhesion [7,51].

Moreover, as it is widely evidenced in the literature [39,57], the low adhesion of the ice is strongly related to the low CAH: in particular, the hysteresis of SLS kept a low value even at low a temperature, and this gave rise to the low ice adhesion.

\subsection{Durability of Wettability Properties}

The results of the wettability after aging in harsh environments are reported in Figures 6-8 (for more details, see the Supplementary Materials).

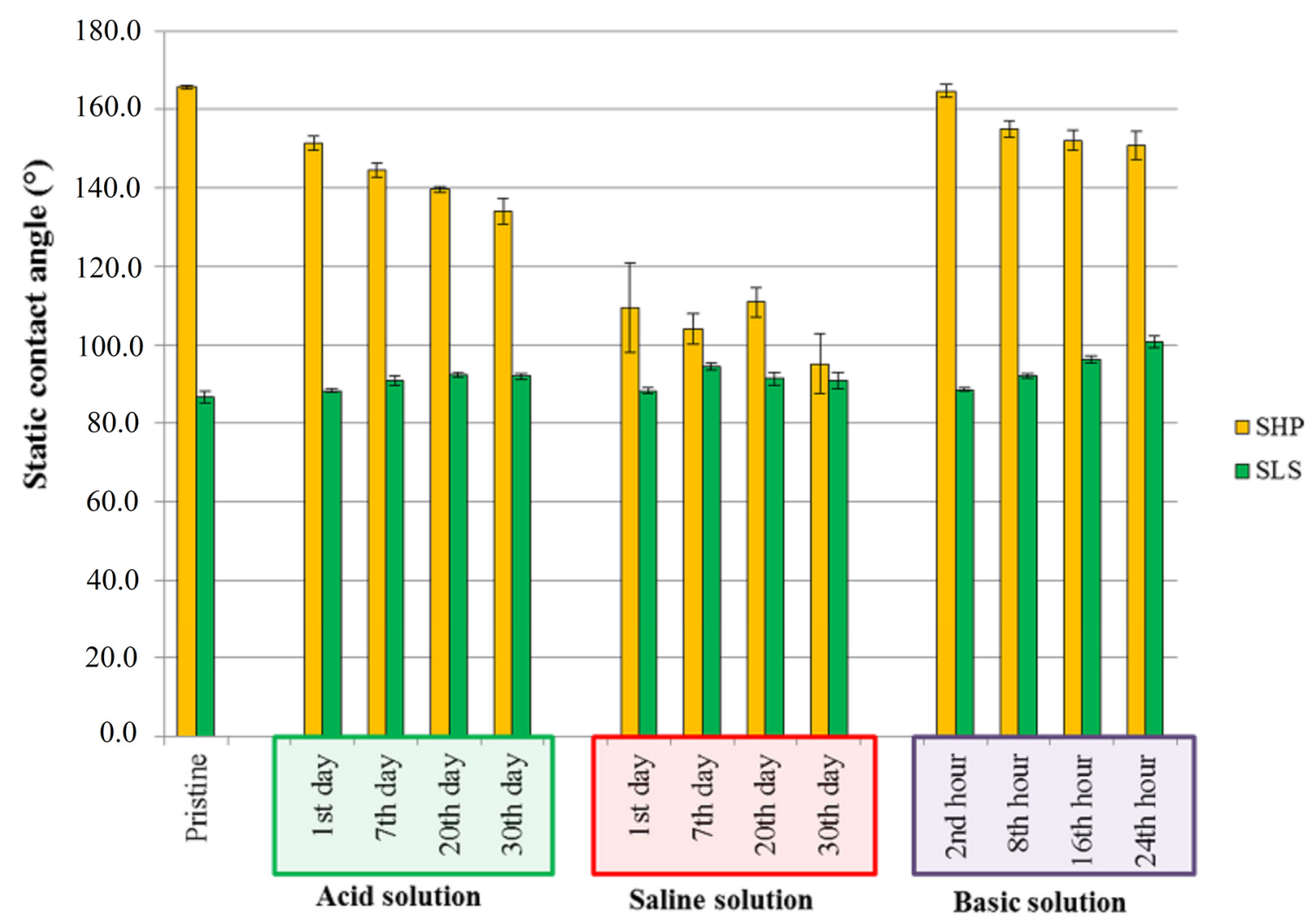

Figure 6. Static contact angles of SHP and SLS after aging in acid, saline and basic solutions. 


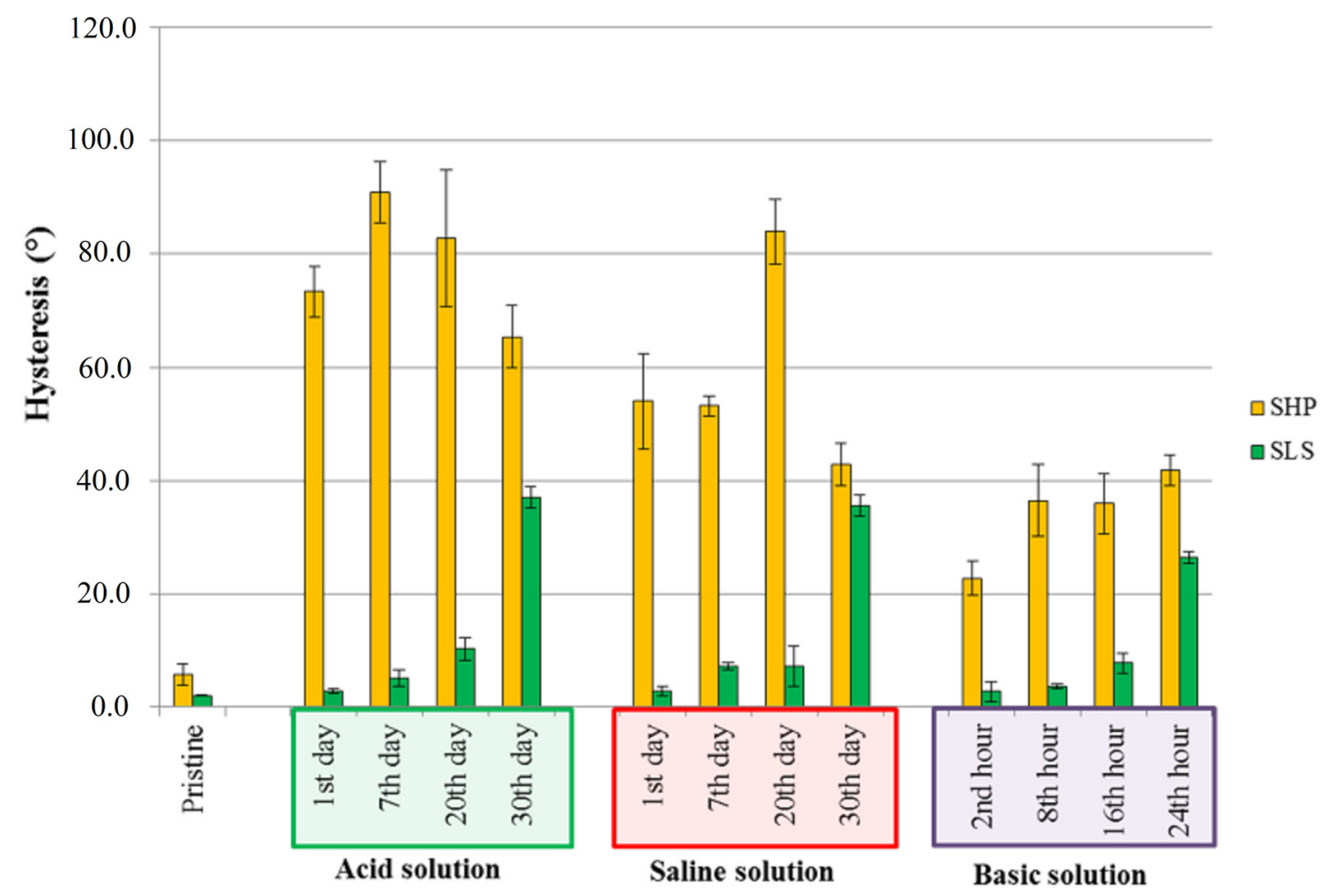

Figure 7. Hysteresis of SHP and SLS after aging in acid, saline and basic solutions.

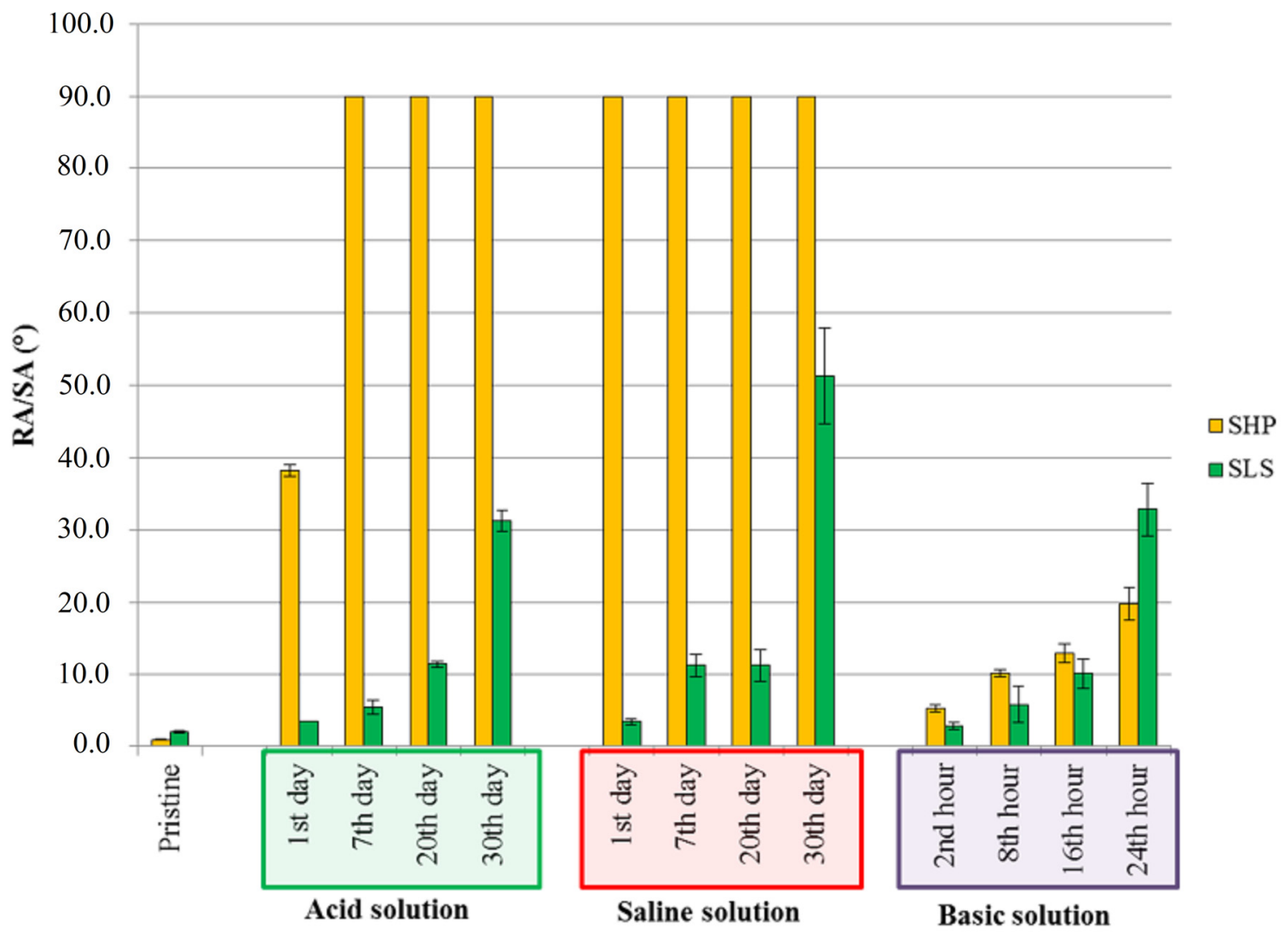

Figure 8. Roll-off/slide-off angles of SHP and SLS after aging in acid, saline and basic solutions: values of $90^{\circ}$ mean that the drops did not roll off. 
As a general consideration, the hysteresis and the roll-off or slide-off angle are strongly affected by the degradation of the coatings, and the increase of their values is a clue of the deterioration of the surfaces [58].

In general, the SLS sample showed better durability than the SHP surface: the CAH and SA values of SLS slowly degraded in harsh environments. In acid and saline conditions, they remained substantially steady after 20 days, while a sudden degradation is observed for SHP surfaces. The test in basic solution is the most critical for both samples as the timescale of degradation is particularly rapid. Anyhow, the samples maintained a rather good RA/SA value (about $10^{\circ}$ ) up to $16 \mathrm{~h}$.

After the exposition in wet chemical solutions, the FT-IR spectra of the samples evidence some differences with respect to the pristine ones (Figure 9).
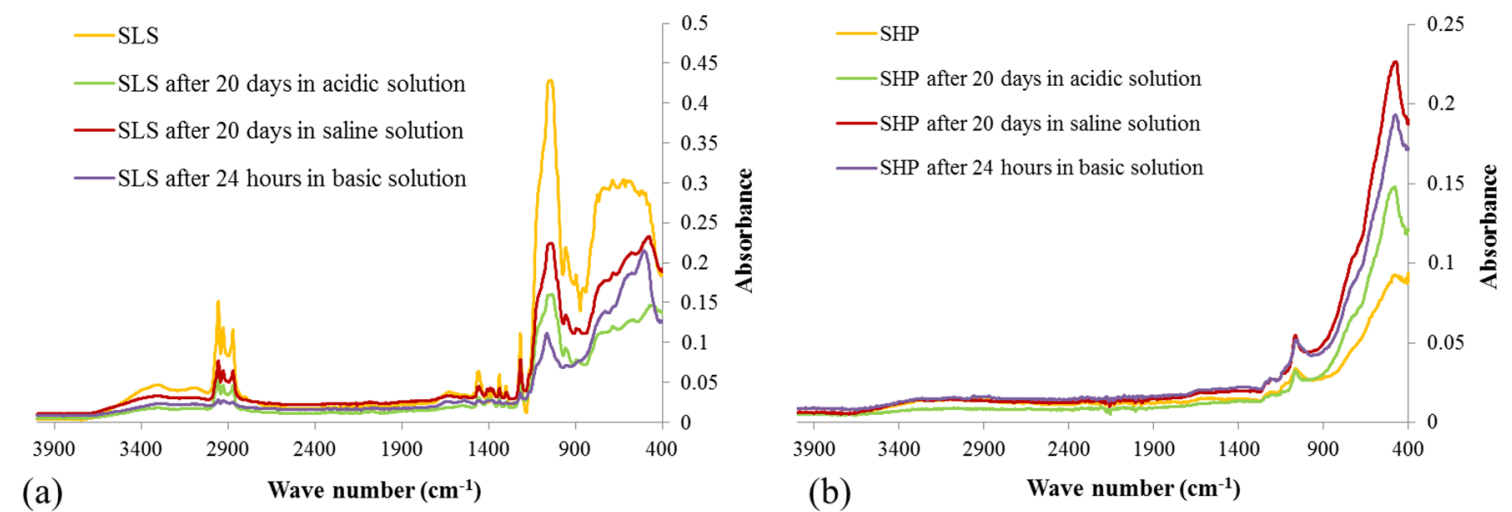

Figure 9. FT-IR of SLS (a) and SHP (b) surfaces after aging in harsh environment.

A degradation of the SLS due to the Dyn-50 layer reduction after the aging tests is visible from the decreasing intensity of the peaks around $2900 \mathrm{~cm}^{-1}$ (aliphatic C-H bonds stretching), whereas the samples aged in acid and saline solutions (Figure 9a, green and red lines, respectively) showed only a smaller intensity reduction. These results are in good agreement with the wettability analysis of the aged samples.

The FT-IR spectra of SHP samples are less explicative because of the very weak signals of the FAS layer coating. Anyhow, the signals from the underlying pseudo-boehmite layer are well visible (780-470 $\mathrm{cm}^{-1}$ ) and their increasing intensities after aging point out the loss of the FAS, especially severe for the basic solution, if considering the short immersion times (Figure 9b).

All the collected data evidenced that the loss of the wettability properties is due in both cases to the significant depletion of the siloxane coatings. According to the literature, this could be attributed to the hydrolysis reaction of $\mathrm{Si}-\mathrm{O}-\mathrm{Al}$ bonds $[50,59,60]$ catalyzed by acids, bases or nucleophilic chlorine ions. In the SHP, this reaction is likely to occur forthwith because the FAS monolayer is widely exposed to the reacting agents and because the fluorinated chains increase the electrophilic nature of silicon atoms. On the contrary, in SLS, this reaction is strongly hindered by the thick coating that protects the interface: the $\mathrm{Si}-\mathrm{O}-\mathrm{Al}$ bonds are exposed to the water only after the hydrolysis of $\mathrm{Si}-\mathrm{O}-\mathrm{Si}$ bonds occurs. Furthermore, the better durability of SLS with respect to SHP can be attributed to the thicker layer of Dyn-50 deposed on the substrate, not only for its role in protecting the interface but also because it has a kind of self-healing properties. When the first functionalized Si-O-Si layer is hydrolyzed, a second layer equal to the first is still available to ensure the wettability properties. In order to confirm this, a durability study on the SLS-1, which is coated with a layer of Dyn-50 of the same order of thickness as SLS, was carried out. As expected, better durability of this sample with respect to the SHP was evidenced for the acid and saline solutions, while the basic solution rapidly degraded the sample. Data highlighting the differences in surface deterioration are reported in Table 3. Moreover, by comparing the hysteresis and sliding angles of SLS and SLS-1, a better endurance of the SLS sample is shown, especially for the tests in acid ad saline solutions. This confirms that the pseudo-boehmite 
layer, grafting the coating with $\mathrm{Si}-\mathrm{O}-\mathrm{Al}$ chemical bonds, gives rise to a more durable surface with respect to the mere material deposition on the substrate.

Table 3. Significant wettability results after durability test in wet chemicals for SLS, SLS-1 and SHP.

\begin{tabular}{|c|c|c|c|c|c|c|c|c|c|}
\hline \multirow{3}{*}{ Sample } & \multicolumn{3}{|c|}{ Acid Solution } & \multicolumn{3}{|c|}{ Saline Solution } & \multicolumn{3}{|c|}{ Basic Solution } \\
\hline & \multicolumn{3}{|c|}{7 Days } & \multicolumn{3}{|c|}{15 Days } & \multicolumn{3}{|c|}{$24 \mathrm{~h}$} \\
\hline & $\begin{array}{l}\text { CA } \\
\left({ }^{\circ}\right)\end{array}$ & $\begin{array}{c}\text { CAH } \\
\left({ }^{\circ}\right)\end{array}$ & $\begin{array}{c}\text { RA/SA } \\
\left({ }^{\circ}\right)\end{array}$ & $\begin{array}{l}\text { CA } \\
\left(^{\circ}\right)\end{array}$ & $\begin{array}{c}\text { CAH } \\
\left({ }^{\circ}\right)\end{array}$ & $\begin{array}{c}\text { RA/SA } \\
\left({ }^{\circ}\right)\end{array}$ & $\begin{array}{l}\text { CA } \\
\left({ }^{\circ}\right)\end{array}$ & $\begin{array}{c}\text { CAH } \\
\left({ }^{\circ}\right)\end{array}$ & $\begin{array}{c}\text { RA/SA } \\
\left({ }^{\circ}\right)\end{array}$ \\
\hline SLS & 89.1 & 6.7 & 6.1 & 92.8 & 6.6 & 8.2 & 100.6 & 26.4 & 32.8 \\
\hline SLS-1 & 88.6 & 26.3 & 44.5 & 99.5 & 38.9 & 56.9 & 99.4 & 33.4 & 39.8 \\
\hline SHP & 144.6 & 90.9 & no roll & 87.1 & 46.2 & no roll & 150.9 & 41.7 & 19.7 \\
\hline
\end{tabular}

The durability of the slippery sample is comparable to that of some SLIPS described in the literature [22,24]; the remarkable stability of the SLS surface evidenced also a role as a protective layer for the aluminum substrate under the three tested aging environments. This behavior is considered very important for prospective applications in outdoor environments.

\subsection{Durability of Ice-Phobic Properties}

Shear stress tests were repeated twenty times on the same bars for sample SLS and compared to the results obtained for SHP after the same test procedure [12]. The shear stress values are shown in Figure 10.

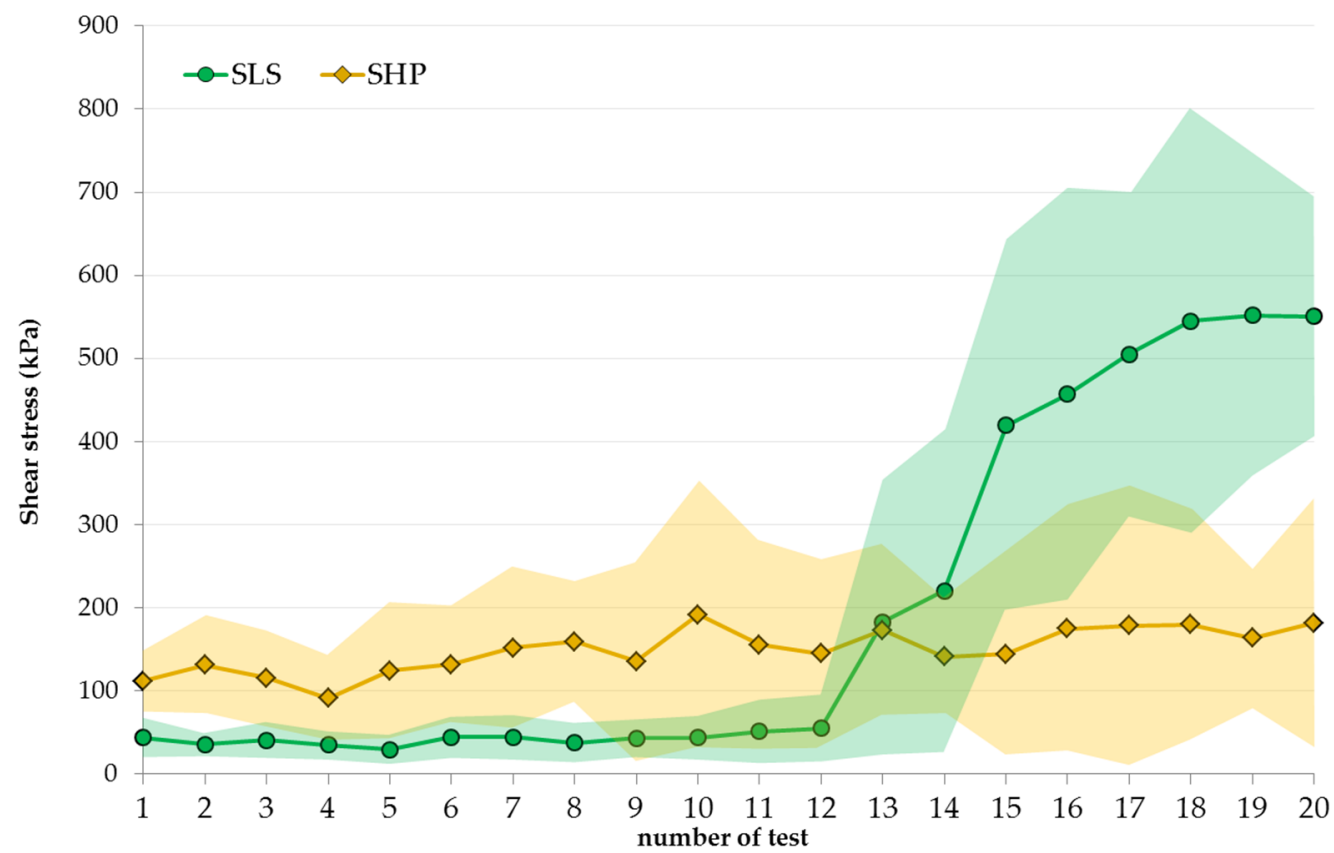

Figure 10. Durability of ice-phobic properties of SLS and SHP with repeated shear stress measurements. Pale areas represent the standard deviation for each value.

As reported in a previous study, the SHP evidenced remarkable durability for 20 shear stress cycles and showed better performances with respect to several superhydrophobic coatings described in the literature $[12,61]$. This behavior is thought to be attributable to the presence of the micro-roughness which enhances the hardness of the sample that is, consequently, less exposed to ice abrasion. In order to confirm this, the hardness of a sandblasted and of a pristine aluminum alloy sample was tested with a microhardness tester and the resulting marks were measured by SEM analysis. The hardness of the pristine aluminum was calculated to be about $70 \mathrm{hv}$, while on the sandblasted sample, no indentation 
was evidenced, and this could indicate harder material. For comparison purposes, we measured a tumbled finished specimen, assuming that the sandblasting treatment could give rise to a surface with similar mechanical properties. The hardness was about $169 \mathrm{hv}$ and we can assume that the sandblasted specimen should have a similar or higher value.

The SLS evidenced a very good and constant ice-phobic behavior for as long as 12 tests, after which the degradation began to appear, followed by a strong and sudden increase of the ice adhesion during the further tests. This trend can be attributed to the progressive depletion of Dyn-50 layers up to the complete removal of the coating, with a consequent abrupt surge of ice adhesion. The proposed mechanism of the coating depletion is double: the breakage of the $\mathrm{Si-O}-\mathrm{Si}$ bonds is due to the reaction with water during the process of ice formation $[50,59,60]$, similarly to what was mentioned for wet chemical tests (Section 3.4) and the mechanical abrasion of the coating layer due to the ice during the process of ice detachment.

The partial removal of the coating from the surface also exposes the acicular nanolayer to the ice and the nanostructures indented into the ice are very likely to be damaged during ice removal $[3,9,50,62]$. Further confirmation of this comes from SEM images and EDX analysis of SLS rods after 20 shear stress tests (Figure 11). In contrast with the pristine sample (Figure 2), the pseudo-boehmite layer is visible but it is inhomogeneous: it clearly comes out in some zones (Figure 11a) but appears less porous and not nanostructured in others (Figure 11b). This is attributed to ice friction. Moreover, from the EDX, it is confirmed that the Dyn-50 is almost completely removed (Si content $<1$ at. \%).

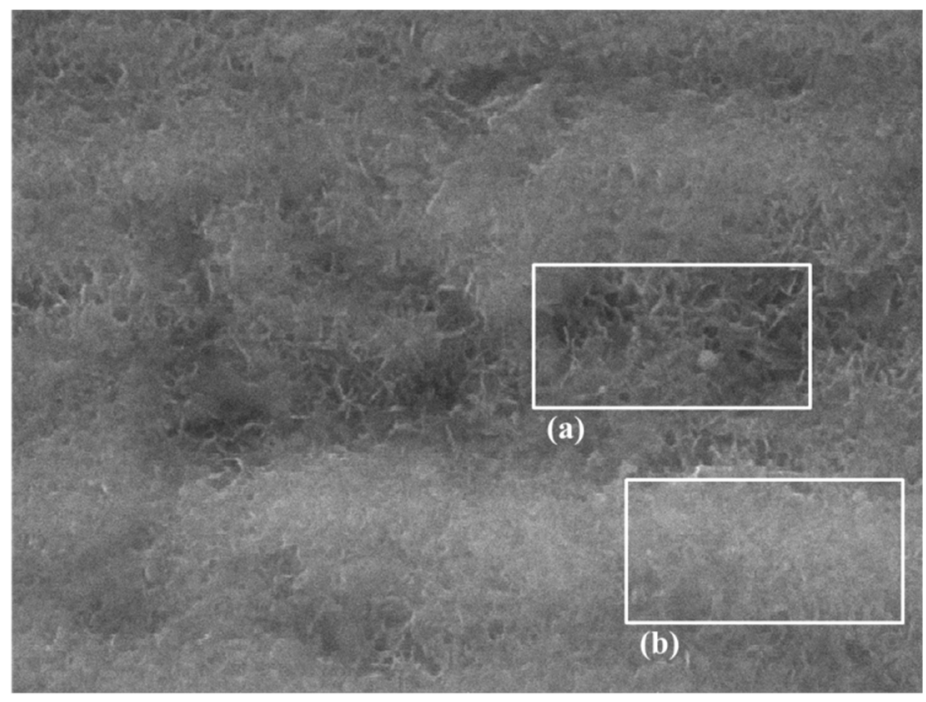

$2 \mu \mathrm{m}$

Figure 11. SEM image of SLS rod after 20 shear stress tests: the boxes evidenced (a) acicular nano layer and (b) highly deteriorated oxide layer.

\section{Conclusions}

In this study, we present an economical, environmentally friendly and fast process to synthetize a dry and fluorine-free slippery aluminum surface with ice-phobic properties. A comparison between a superhydrophobic coated aluminum (SHP) and the slippery surface (SLS) was carried out. They evidenced similar anti-icing behavior in lowering the nucleation temperature of freezing water; however, the SLS demonstrated a lower adhesion to the ice. Durability tests were carried out both on hydrophobic and ice-phobic behavior: SLS maintains its wettability properties in wet chemicals for a long time (up to 20 days) while SHP completely lost the super-hydrophobicity in few hours in all the tested environments. On the contrary, SHP demonstrated longer ice-phobic durability (up to twenty repeated shear stress tests) than SLS, which lost the ice-phobic property after 12 tests. The very good 
durability of ice-phobicity is related to the hardness of the SHP, enhanced by the sandblasting process. On the other side, the SLS evidenced a loss of coating and a deterioration of the nanostructure.

SLS surfaces are promising materials because of their very good ice-phobic and anti-icing properties and remarkable durability in harsh environments even if further study is necessary in order to enhance their ice-phobic durability. A three-step process combining the sandblasting, the hydrothermal treatment and the Dyn-50 coating could lead to a better ice-phobic durability, although an increased surface area is expected to slightly enhance the adhesion between the sample and the ice. A deeper understanding of these conflicting effects (enhanced durability vs enhanced ice adhesion) is crucial to achieving a more durable ice-phobic surface.

Supplementary Materials: The following are available online at http://www.mdpi.com/2079-6412/10/2/111/s1, Figure S1: home-made equipment for shear stress measures, Table S1: Protocol for shear stress measures, Figure S2: sample treatment protocol for the durability testing in wet chemicals, Table S2: Schedule of the wettability measures after the immersion in wet chemicals, Figure S3: durability in acid solution: static contact angles, Figure S4: durability in acid solution: hysteresis, Figure S5: durability in acid solution: roll-off and sliding angles. Figure S6: durability in saline solution: static contact angles, Figure S7: durability in saline solution: hysteresis angles, Figure S8: durability in saline solution: roll-off and sliding angles.

Author Contributions: Conceptualization, M.B. and G.S.d.M.; Methodology, M.B. and G.S.d.M.; Validation, G.S.d.M.; Formal Analysis, G.S.d.M.; Investigation, M.B., C.C. and G.S.d.M.; Data Curation, M.B.; Writing-Original Draft Preparation, M.B., C.C. and G.S.d.M.; Writing-Review and Editing, M.B., C.C. and G.S.d.M.; Supervision, M.B. and C.C.; Project Administration, C.C. All authors have read and agreed to the published version of the manuscript.

Funding: This research was funded by the Research Fund for the Italian Electrical System in compliance with the Decree of Minister of Economic Development on 16 April 2018.

Acknowledgments: The authors would like to thank Nella Belgiovine, Stefano Capelli, Gian Paolo Carbi, Emanuela Erbaldi, Francesco Pini and Flavia Tulli, for their supporting activity.

Conflicts of Interest: The authors declare no conflict of interest.

\section{References}

1. Latthe, S.S.; Sutar, R.S.; Bhosale, A.K.; Nagappan, S.; Ha, C.-S.; Sadasivuni, K.K.; Liu, S.; Xing, R. Recent developments in air-trapped superhydrophobic and liquid-infused slippery surfaces for anti-icing application. Prog. Org. Coat. 2019, 137, 105373. [CrossRef]

2. Farzaneh, M.; Gauthier, H.; Castellana, G.; Engelbrecht, C.; Elìasson, Á.J.; Fikke, S.M.; Greyling, C.; Gutman, I.; Hayashi, T.; Jakl, F. Coatings for Protecting Overhead Power Network Equipment in Winter Conditions; CIGRE: Paris, France, 2015; ISBN 978-2-85873-334-7.

3. Farhadi, S.; Farzaneh, M.; Kulinich, S.A. Anti-icing performance of superhydrophobic surfaces. Appl. Surf. Sci. 2011, 257, 6264-6269. [CrossRef]

4. Cao, L.; Jones, A.K.; Sikka, V.K.; Wu, J.; Gao, D. Anti-icing superhydrophobic coatings. Langmuir 2009, 25, 12444-12448. [CrossRef] [PubMed]

5. Jung, S.; Dorrestijn, M.; Raps, D.; Das, A.; Megaridis, C.M.; Poulikakos, D. Are superhydrophobic surfaces best for icephobicity? Langmuir 2011, 27, 3059-3066. [CrossRef] [PubMed]

6. Narhe, R.D.; Beysens, D.A. Growth dynamics of water drops on a square-pattern rough hydrophobic surface. Langmuir 2007, 23, 6486-6489. [CrossRef]

7. Chen, J.; Liu, J.; He, M.; Li, K.; Cui, D.; Zhang, Q.; Zeng, X.; Zhang, Y.; Wang, J.; Song, Y. Superhydrophobic surfaces cannot reduce ice adhesion. Appl. Phys. Lett. 2012, 101, 111603. [CrossRef]

8. Varanasi, K.K.; Deng, T.; Smith, J.D.; Hsu, M.; Bhate, N. Frost formation and ice adhesion on superhydrophobic surfaces. Appl. Phys. Lett. 2010, 97, 234102. [CrossRef]

9. Kulinich, S.A.; Farhadi, S.; Nose, K.; Du, X.W. Superhydrophobic surfaces: Are they really ice-repellent? Langmuir 2011, 27, 25-29. [CrossRef]

10. Verho, T.; Bower, C.; Andrew, P.; Franssila, S.; Ikkala, O.; Ras, R.H.A. Mechanically durable superhydrophobic surfaces. Adv. Mater. 2011, 23, 673-678. [CrossRef]

11. Boinovich, L.B.; Emelyanenko, A.M.; Emelyanenko, K.A.; Modin, E.B. Modus operandi of protective and anti-icing mechanisms underlying the design of longstanding outdoor icephobic coatings. ACS Nano 2019, 13, 4335-4346. [CrossRef] 
12. Balordi, M.; Cammi, A.; Santucci de Magistris, G.; Chemelli, C. Role of micrometric roughness on anti-ice properties and durability of hierarchical super-hydrophobic aluminum surfaces. Surf. Coat. Technol. 2019, 374, 549-556. [CrossRef]

13. Occurrence and use of Highly Fluorinated Substances and Alternatives; 7/15; Swedish Chemical Agency: Stockholm, Sweden, 2015; p. 112.

14. Kreder, M.J.; Alvarenga, J.; Kim, P.; Aizenberg, J. Design of anti-icing surfaces: Smooth, textured or slippery? Nat. Rev. Mater. 2016, 1, 15003. [CrossRef]

15. Wang, N.; Xiong, D.; Lu, Y.; Pan, S.; Wang, K.; Deng, Y.; Shi, Y. Design and fabrication of the lyophobic slippery surface and its application in anti-icing. J. Phys. Chem. C 2016, 120, 11054-11059. [CrossRef]

16. Kim, P.; Wong, T.-S.; Alvarenga, J.; Kreder, M.J.; Adorno-Martinez, W.E.; Aizenberg, J. Liquid-infused nanostructured surfaces with extreme anti-ice and anti-frost performance. ACS Nano 2012, 6, 6569-6577. [CrossRef] [PubMed]

17. Wong, T.-S.; Kang, S.H.; Tang, S.K.Y.; Smythe, E.J.; Hatton, B.D.; Grinthal, A.; Aizenberg, J. Bioinspired self-repairing slippery surfaces with pressure-stable omniphobicity. Nature 2011, 477, 443-447. [CrossRef]

18. Liu, Q.; Yang, Y.; Huang, M.; Zhou, Y.; Liu, Y.; Liang, X. Durability of a lubricant-infused electrospray silicon rubber surface as an anti-icing coating. Appl. Surf. Sci. 2015, 346, 68-76. [CrossRef]

19. Smith, J.D.; Dhiman, R.; Anand, S.; Reza-Garduno, E.; Cohen, R.E.; McKinley, G.H.; Varanasi, K.K. Droplet mobility on lubricant-impregnated surfaces. Soft Matter 2013, 9, 1772-1780. [CrossRef]

20. Lee, H.; Alcaraz, M.L.; Rubner, M.F.; Cohen, R.E. Zwitter-wettability and antifogging coatings with frost-resisting capabilities. ACS Nano 2013, 7, 2172-2185. [CrossRef]

21. Zhang, J.; Gu, C.; Tu, J. Robust slippery coating with superior corrosion resistance and anti-icing performance for az31b mg alloy protection. ACS Appl. Mater. Interfaces 2017, 9, 11247-11257. [CrossRef]

22. Zhang, M.; Yu, J.; Chen, R.; Liu, Q.; Liu, J.; Song, D.; Liu, P.; Gao, L.; Wang, J. Highly transparent and robust slippery lubricant-infused porous surfaces with anti-icing and anti-fouling performances. J. Alloys Compd. 2019, 803, 51-60. [CrossRef]

23. Nguyen, T.-B.; Park, S.; Jung, Y.; Lim, H. Effects of hydrophobicity and lubricant characteristics on anti-icing performance of slippery lubricant-infused porous surfaces. J. Ind. Eng. Chem. 2019, 69, 99-105. [CrossRef]

24. Veronesi, F.; Boveri, G.; Raimondo, M. Amphiphobic nanostructured coatings for industrial applications. Materials 2019, 12, 787. [CrossRef] [PubMed]

25. Jafari, R.; Farzaneh, M. Fabrication of superhydrophobic nanostructured surface on aluminum alloy. Appl. Phys. A 2011, 102, 195-199. [CrossRef]

26. Michael, N.; Bhushan, B. Hierarchical roughness makes superhydrophobic states stable. Microelectron. Eng. 2007, 84, 382-386. [CrossRef]

27. Saifaldeen, Z.S.; Khedir, K.R.; Cansizoglu, M.F.; Demirkan, T.; Karabacak, T. Superamphiphobic aluminum alloy surfaces with micro and nanoscale hierarchical roughness produced by a simple and environmentally friendly technique. J. Mater. Sci. 2014, 49, 1839-1853. [CrossRef]

28. Heydari, G.; Tyrode, E.; Visnevskij, C.; Makuska, R.; Claesson, P.M. Temperature-dependent deicing properties of electrostatically anchored branched brush layers of poly(ethylene oxide). Langmuir 2016, 32, 4194-4202. [CrossRef]

29. Malavasi, I.; Bernagozzi, I.; Antonini, C.; Marengo, M. Assessing durability of superhydrophobic surfaces. Surf. Innov. 2015, 3, 49-60. [CrossRef]

30. Geiculescu, A. A microstructural investigation of low-temperature crystalline alumina films grown on aluminum. Thin Solid Films 2003, 426, 160-171. [CrossRef]

31. Rider, A.N. The influence of porosity and morphology of hydrated oxide films on epoxy-aluminium bond durability. J. Adhes. Sci. Technol. 2001, 15, 395-422. [CrossRef]

32. Motta, A.; Cannelli, O.; Boccia, A.; Zanoni, R.; Raimondo, M.; Caldarelli, A.; Veronesi, F. A mechanistic explanation of the peculiar amphiphobic properties of hybrid organic-inorganic coatings by combining xps characterization and dft modeling. ACS Appl. Mater. Interfaces 2015, 7, 19941-19947. [CrossRef]

33. Rio, E.; Boulogne, F. Withdrawing a solid from a bath: How much liquid is coated? Adv. Colloid Interface Sci. 2017, 247, 100-114. [CrossRef] [PubMed]

34. Damayanti, N.P. Preparation of superhydrophobic pet fabric from al2o3-sio2 hybrid: Geometrical approach to create high contact angle surface from low contact angle materials. J. Sol-Gel Sci. Technol. 2010, 56, 47-52. [CrossRef] 
35. Wang, F.; Li, C.; Lv, Y.; Lv, F.; Du, Y. Ice accretion on superhydrophobic aluminum surfaces under low-temperature conditions. Cold Reg. Sci. Technol. 2010, 62, 29-33. [CrossRef]

36. Prysiazhnyi, V. Plasma treatment of aluminum using a surface barrier discharge operated in air and nitrogen: Parameter optimization and related effects. Plasma Sci. Technol. 2013, 15, 794-799. [CrossRef]

37. Niemelä-Anttonen, H.; Koivuluoto, H.; Tuominen, M.; Teisala, H.; Juuti, P.; Haapanen, J.; Harra, J.; Stenroos, C.; Lahti, J.; Kuusipalo, J. Icephobicity of slippery liquid infused porous surfaces under multiple freeze-thaw and ice accretion-detachment cycles. Adv. Mater. Interfaces 2018, 5, 1800828. [CrossRef]

38. Sojoudi, H.; Wang, M.; Boscher, N.D.; McKinley, G.H.; Gleason, K.K. Durable and scalable icephobic surfaces: Similarities and distinctions from superhydrophobic surfaces. Soft Matter 2016, 12, 1938-1963. [CrossRef]

39. Meuler, A.J.; Smith, J.D.; Varanasi, K.K.; Mabry, J.M.; McKinley, G.H.; Cohen, R.E. Relationships between water wettability and ice adhesion. ACS Appl. Mater. Interfaces 2010, 2, 3100-3110. [CrossRef]

40. Li, Q.; Guo, Z. Fundamentals of icing and common strategies for designing biomimetic anti-icing surfaces. J. Mater. Chem. A 2018, 6, 13549-13581. [CrossRef]

41. Zhang, Z.; Liu, X.-Y. Control of ice nucleation: Freezing and antifreeze strategies. Chem. Soc. Rev. 2018, 47, 7116-7139. [CrossRef]

42. He, M.; Wang, J.; Li, H.; Song, Y. Super-hydrophobic surfaces to condensed micro-droplets at temperatures below the freezing point retard ice/frost formation. Soft Matter 2011, 7, 3993. [CrossRef]

43. Zhang, Q.; Jin, B.; Wang, B.; Fu, Y.; Zhan, X.; Chen, F. Fabrication of a highly stable superhydrophobic surface with dual-scale structure and its antifrosting properties. Ind. Eng. Chem. Res. 2017, 56, 2754-2763. [CrossRef]

44. Anand, S.; Paxson, A.T.; Dhiman, R.; Smith, J.D.; Varanasi, K.K. Enhanced condensation on lubricant-impregnated nanotextured surfaces. ACS Nano 2012, 6, 10122-10129. [CrossRef]

45. Zhan, X.; Yan, Y.; Zhang, Q.; Chen, F. A novel superhydrophobic hybrid nanocomposite material prepared by surface-initiated aget atrp and its anti-icing properties. J. Mater. Chem. A 2014, 2, 9390-9399. [CrossRef]

46. Song, J.; Zhao, D.; Han, Z.; Xu, W.; Lu, Y.; Liu, X.; Liu, B.; Carmalt, C.J.; Deng, X.; Parkin, I.P. Super-robust superhydrophobic concrete. J. Mater. Chem. A 2017, 5, 14542-14550. [CrossRef]

47. Wei, C.; Jin, B.; Zhang, Q.; Zhan, X.; Chen, F. Anti-icing performance of super-wetting surfaces from icing-resistance to ice-phobic aspects: Robust hydrophobic or slippery surfaces. J. Alloys Compd. 2018, 765, 721-730. [CrossRef]

48. Bi, Y.; Cabriolu, R.; Li, T. Heterogeneous ice nucleation controlled by the coupling of surface crystallinity and surface hydrophilicity. J. Phys. Chem. C 2016, 120, 1507-1514. [CrossRef]

49. Fu, Q.; Wu, X.; Kumar, D.; Ho, J.W.C.; Kanhere, P.D.; Srikanth, N.; Liu, E.; Wilson, P.; Chen, Z. Development of sol-gel icephobic coatings: Effect of surface roughness and surface energy. ACS Appl. Mater. Interfaces 2014, 6, 20685-20692. [CrossRef]

50. Wang, Y.; Xue, J.; Wang, Q.; Chen, Q.; Ding, J. Verification of icephobic/anti-icing properties of a superhydrophobic surface. ACS Appl. Mater. Interfaces 2013, 5, 3370-3381. [CrossRef]

51. Bharathidasan, T.; Kumar, S.V.; Bobji, M.S.; Chakradhar, R.P.S.; Basu, B.J. Effect of wettability and surface roughness on ice-adhesion strength of hydrophilic, hydrophobic and superhydrophobic surfaces. Appl. Surf. Sci. 2014, 314, 241-250. [CrossRef]

52. Susoff, M.; Siegmann, K.; Pfaffenroth, C.; Hirayama, M. Evaluation of icephobic coatings—screening of different coatings and influence of roughness. Appl. Surf. Sci. 2013, 282, 870-879. [CrossRef]

53. Sojoudi, H.; Arabnejad, H.; Raiyan, A.; Shirazi, S.A.; McKinley, G.H.; Gleason, K.K. Scalable and durable polymeric icephobic and hydrate-phobic coatings. Soft Matter 2018, 14, 3443-3454. [CrossRef] [PubMed]

54. Juuti, P.; Haapanen, J.; Stenroos, C.; Niemelä-Anttonen, H.; Harra, J.; Koivuluoto, H.; Teisala, H.; Lahti, J.; Tuominen, M.; Kuusipalo, J.; et al. Achieving a slippery, liquid-infused porous surface with anti-icing properties by direct deposition of flame synthesized aerosol nanoparticles on a thermally fragile substrate. Appl. Phys. Lett. 2017, 110, 161603. [CrossRef]

55. Coady, M.J.; Wood, M.; Wallace, G.Q.; Nielsen, K.E.; Kietzig, A.-M.; Lagugné-Labarthet, F.; Ragogna, P.J. Icephobic behavior of uv-cured polymer networks incorporated into slippery lubricant-infused porous surfaces: Improving slips durability. ACS Appl. Mater. Interfaces 2018, 10, 2890-2896. [CrossRef] [PubMed]

56. Ruan, M.; Li, W.; Wang, B.; Deng, B.; Ma, F.; Yu, Z. Preparation and anti-icing behavior of superhydrophobic surfaces on aluminum alloy substrates. Langmuir 2013, 29, 8482-8491. [CrossRef] [PubMed] 
57. Sarshar, M.A.; Swarctz, C.; Hunter, S.; Simpson, J.; Choi, C.-H. Effects of contact angle hysteresis on ice adhesion and growth on superhydrophobic surfaces under dynamic flow conditions. Colloid Polym. Sci. 2013, 291, 427-435. [CrossRef]

58. Amirfazli, A. Superomniphobic Surfaces for Military Applications: Nano- and Micro-fabrication Methods; Defence Research and Development Canada: Ottawa, ON, Canada, 2011; p. 130.

59. Boinovich, L.B.; Emelyanenko, A.M.; Ivanov, V.K.; Pashinin, A.S. Durable icephobic coating for stainless steel. ACS Appl. Mater. Interfaces 2013, 5, 2549-2554. [CrossRef] [PubMed]

60. Kulinich, S.A.; Honda, M.; Zhu, A.L.; Rozhin, A.G.; Du, X.W. The icephobic performance of alkyl-grafted aluminum surfaces. Soft Matter 2015, 11, 856-861. [CrossRef]

61. Momen, G.; Jafari, R.; Farzaneh, M. Ice repellency behaviour of superhydrophobic surfaces: Effects of atmospheric icing conditions and surface roughness. Appl. Surf. Sci. 2015, 349, 211-218. [CrossRef]

62. Lazauskas, A.; Guobienè, A.; Prosyčevas, I.; Baltrušaitis, V.; Grigaliūnas, V.; Narmontas, P.; Baltrusaitis, J. Water droplet behavior on superhydrophobic sio2 nanocomposite films during icing/deicing cycles. Mater. Charact. 2013, 82, 9-16. [CrossRef]

(C) 2020 by the authors. Licensee MDPI, Basel, Switzerland. This article is an open access article distributed under the terms and conditions of the Creative Commons Attribution (CC BY) license (http://creativecommons.org/licenses/by/4.0/). 